\title{
SPION and doxorubicin-loaded polymeric nanocarriers for glioblastoma theranostics
}

Edurne Luque-Michel ${ }^{\mathrm{a}, \mathrm{b}}$, Laurent Lemaire ${ }^{\mathrm{c}, \mathrm{d}^{*}}$ and Maria J. Blanco-Prieto ${ }^{\mathrm{a}, \mathrm{b}^{*}}$

${ }^{a}$ Department of Pharmacy and Pharmaceutical Technology, School of Pharmacy, University of Navarra, C/Irunlarrea 1, E-31008 Pamplona, Spain

${ }^{b}$ Instituto de Investigación Sanitaria de Navarra (IdiSNA), Pamplona, Spain

'INSERM UMR-S 1066- CNRS 6021, Micro et Nanomédecines Translationnelles - MINT Université d'Angers, 4 rue Larrey, Angers 49933, France

${ }^{d}$ PRISM - Plateforme de Recherche en Imagerie et Spectroscopie Métabolique, Université d'Angers, 4 rue Larrey, Angers 49933, France

Corresponding authors*:

María J. Blanco-Prieto and Laurent Lemaire

\section{GRAPHICAL ABSTRACT}

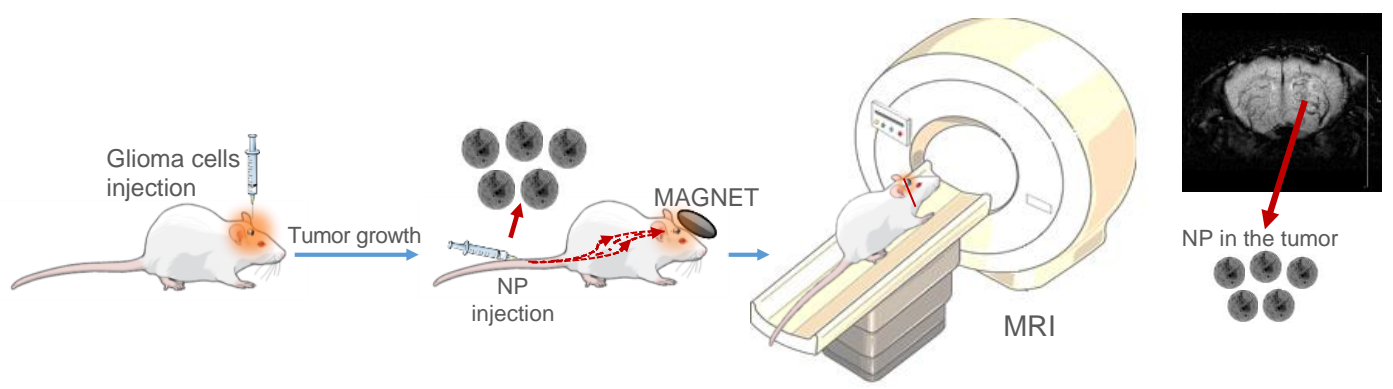

\begin{abstract}
Glioma is a type of cancer with a very poor prognosis with a survival of around 15 months in the case of glioblastoma multiforme (GBM). In order to advance in personalized medicine, we developed polymeric nanoparticles (PNP) loaded with both SPION (Superparamagnetic Iron Oxide Nanoparticles) and doxorubicin (DOX). The former being used for its potential to accumulate the PNP in the tumor under a strong magnetic field and the later for its therapeutic potential. The emulsion solvent and evaporation method was selected to develop monodisperse PNP with high loading efficiency in both SPION and DOX. Once injected in mice, a significant accumulation of the PNP was observed within the tumoral tissue under static magnetic field as observed by MRI leading to a reduction of tumor growth rate.
\end{abstract}

KEYWORDS: Tween 80, MRI, tumor doubling time, glioma, U87-MG, PLGA, SPION

Drug Deliv Transl Res 2021 Jan 6. doi: 10.1007/s13346-020-00880-8.

PMID: 33405212 


\section{INTRODUCTION}

Glioblastoma (GBM) is the most common and the most malignant variant in the wide spectrum of intrinsic glial brain tumors [1]. Morphologically, GBM is highly heterogeneous, diffuse and infiltrative in nature, which makes surgical removal particularly difficult [2]. In parallel, the failure of chemotherapy to reach the brain, as it is protected by the dynamic Blood Brain Barrier (BBB), and the presence of stem cells lead to tumor recurrence [2,3]. In the area of cancer nanomedicine, drugs are encapsulated inside nanocarriers that provide controlled release of the drug once in the tumor. In this sense, nanoparticles (NP) designed to achieve BBB uptake could increase the drug concentration in glioma and then increase the effectiveness of treatment. This is the case of polymeric nanoparticles (PNP) made with surfactants like Tween 80 (T80), which have received increasing attention over the last few years. Widely accepted in the literature, this strategy takes advantage of the adsorption of apolipoproteins across blood plasma onto the T80 NP surface, promoting recognition by LDL receptors in the brain capillary endothelial cells [4-6]; moreover, both neurons and glial cells express LDL receptors [7]. Then, nanomedicine could help antineoplastic drugs not able to cross the BBB naturally to reach the glioma. This is the case of DOX, an antineoplastic agent widely used in the treatment of various cancers which, although toxic against glioma cells, does not cross the BBB [8-10]. Above that, theragnosis, defined as any material that combines the modalities of therapy and imaging into a single package [11], is emerging as a very promise strategy to image and treat brain cancers [12]. In this work, we suggest an association of doxorubicin (DOX) and SPION (superparamagnetic iron oxide nanoparticles) within T80-coated PNP. SPION were selected since they can be monitored non-invasively by magnetic resonance imaging (MRI) and, moreover, they can be targeted to tumor with the use of magnets [13,14]. These theranostic SPION-DOX PNP thus reflect the real-time characteristics of the tumor in each patient and could allow for earlier disease detection, more accurate prognostic information and an enhanced ability to monitor the efficacy of treatment [15]. Besides, the evaluation of the NP accumulation in healthy tissues would allow us to assess the risk of patients developing off-target side effects or to screen patients who are likely to respond positively to the treatment [16].
Specifically, the SPION-DOX-NP developed were injected intravenously in mice, and their ability to reach the GBM under the influence of an external magnetic field was evaluated.

\section{METHODS}

\section{SPION-DOX PNP synthesis and characterization}

SPION-DOX PNP were formulated by the simple emulsion and solvent evaporation method as described before [10]. Briefly, the SPION were covered with oleic acid $(10 \mathrm{mg}$ of iron $/ \mathrm{mL}$ of oleic acid) by 24 hours incubation with continuous shaking, and after that, they were washed and dissolved in dichloromethane. Separately, $0.5 \mathrm{mg}$ of DOX was dissolved overnight in $0.8 \mathrm{~mL}$ of a mixture of triethylamine and ethyl acetate in the proportion 1: 1000. The follow day, $50 \mathrm{mg}$ of polymer poly (Lactic-coGlycolic Acid) (PLGA) (Resomer® RG 503H, PLGA 50:50) were dissolved in the same solution of DOX and $0.2 \mathrm{~mL}$ of SPION covered with oleic acid were added. All of this was poured into $2 \mathrm{~mL}$ of $1 \%$ T80 and sonicated for 20 seconds at 20 Watts in a Microson Ultrasonic Cell Disruptor XL (Branson sonifier 450, Branson Ultrasonics corp., EEUU). Next, the sonicated solution was poured into an aqueous solution of $0.3 \%$ T 80 and $0.4 \%$ polyvinyl alcohol (PVA) with continuous shaking for 1.5 hours. Once the organic solvent had evaporated, the formulation was washed three times (centrifugations at $17000 \mathrm{~g}$ for $10 \mathrm{~min}$ at $4^{\circ} \mathrm{C}$ ) and lyophilized along with $37 \%(\mathrm{w} / \mathrm{w}$ with respect to the amount of PLGA) of the cryoprotector trehalose.

SPION-DOX PNP size and surface charge were characterized using a Zetasizer Nano ZS (Malvern Instruments, UK), and the size of the NP was measured by TEM images. The DOX loading efficiency was determined fluorimetrically in a Tecan GENios microplate reader (Tecan Group Ltd, Maennedorf, Switzerland) at an excitation and emission wavelength of 485 and $580 \mathrm{~nm}$. In addition to this, the encapsulation of SPION was calculated and analyzed spectrophotometrically $(\lambda=300$ $\mathrm{nm}$ ) using a microplate PowerWave XS Microplate Spectrophotometer (BioTek). Fluorescence and absorbance were converted into $\mu \mathrm{g} / \mathrm{mL}$ DOX or SPION using a calibration curve previously set.

\section{In vivo studies}


A scheme of the in vivo studies performed is presented in the Fig 1.

All institutional and national guidelines for the care and use of laboratory animals were followed. Animal care and use were in accordance with the regulations of the French Ministry of Agriculture and approved by the Pays de la Loire Ethics in Animal Experimentation Committee under project number 01858.03.

\subsection{Intracranial inoculation of GBM cells}

Tumor implantation was performed via stereotaxic inoculation of the human U87 glioma cells in 8-week-old female nude mice. As described previously [17], mice were anesthetized with Xylazine/Ketamine (50/30 UI; $20 \mu \mathrm{L} / \mathrm{g}$ ) and placed in a stereotaxic holder with a heating pad to maintain the appropriate physiological temperature. First, a hole in the skull was drilled to, secondly, inject $4 \mu \mathrm{L}$ (at 0.5 $\mathrm{uL} / \mathrm{min}$ ) suspension of $8 \times 10^{4}$ glioma cells, on the fixed coordinates according to the bregma: $0.5 \mathrm{~mm}$ anterior, $2.5 \mathrm{~mm}$ right lateral, and $4 \mathrm{~mm}$ depth. After surgery, mice received a single 30 $\mu \mathrm{g} / \mathrm{kg}$ subcutaneous injection of Buprecare (buprenorphin). In vivo experiments were performed 9-10 days later, after checking by MRI that the tumor had developed. The different groups of mice were formed maintaining an equitable distribution of tumor sizes.

\subsection{Magnetic targeting and MRI monitoring}

To assess the magnetic and contrast capability of SPION-DOX PNP, 12 tumor-bearing animals were equitably divided into two treatment groups: NP targeted using a magnet $(n=6)$ and NP without magnetic targeting $(n=6)$. Each group received intravenously $16 \mathrm{mg} / \mathrm{kg}$ of $\mathrm{Fe}$ and 5 $\mathrm{mg} / \mathrm{kg}$ of DOX in SPION-DOX PNP synthetized with surfactant $\mathrm{T} 80$ and reconstituted in physiological serum $(0.2 \mathrm{~mL})$. As previously described [18], an external 0.4-T (190 Tm${ }^{-1}$ magnetic field gradient) targeting magnetic field, $8 \mathrm{~mm}$ diameter/4 $\mathrm{mm}$ high disk-shaped neodymium magnets (Supermagnete, Gottmadingen, Germany) were placed onto the top of the head of the mice for 1 hour. During the MR protocol, mice were anesthetized with $0.5 \%$ isoflurane and respiration was monitored. Furthermore, animal body temperature was maintained throughout the experiment at 36.5-37.5 ${ }^{\circ} \mathrm{C}$ by using a feedback-regulated heating pad.
MRI was performed using a 7T scanner (Biospec 70/20 Avance III, Bruker Wissembourg, France) equipped with BGA12S gradient system (675 $\mathrm{mT} / \mathrm{m})$. Prior to injection, animals were controlled to assess tumor sizes using a Rapid Acquisition with Relaxation Enhancement sequence $(\mathrm{TR}=3200 \mathrm{~ms}$; $\mathrm{TE}=33 \mathrm{~ms}$, RARE factor 4 , matrix size $=256 \times 128 ; \mathrm{FOV}=2 \times 1$ $\mathrm{cm}$, slice thickness $=1 \mathrm{~mm}$ ). A multiple gradient echo image, using the same geometrical parameters, was also used to produce $\mathrm{T} 2 *$ maps prior to injection; susceptibility weighted images (SWI) were also collected before NP injection $(\mathrm{TR}=350 \mathrm{~ms}$; TE=18 ms; Slice thickness $1 \mathrm{~mm}$; matrix size $=384 \times 192 ; \mathrm{FOV}=2 \times 1 \mathrm{~cm})$. Half of each group (3 mice/group) was continuously analyzed by MRI for 2.5 hours and the other half was imaged only 4 hours after the injection. SPION-DOX PNP deposition was qualitatively observed using susceptibility weighted images (SWI) and quantitated from the multiple gradient echo set of images by calculating the relaxation rate of transverse magnetization $(\mathrm{R} 2 *=1 / \mathrm{T} 2 *)$. Since the accumulation of PNP is often heterogeneous, R2* were averaged over the entire tumor.

\subsection{Efficacy studies}

To determine the efficacy of the treatment with SPION-DOX PNP, tumor-bearing mice were treated twice with a time interval of $48 \mathrm{~h}$ by intravenous injection of SPION-DOX PNP (at a DOX dose of $5 \mathrm{mg} / \mathrm{kg}$ ). One group was exposed to $1 \mathrm{~h}$ external magnet targeting after each injection $(n=7)$, while the other was not exposed to the external magnet $(n=5)$. To monitor the efficacy of the treatment, tumor size was measured from the MRI dataset prior to the first PNP injection (day 0), day 2, day 5, and day 7 . Volumes were calculated from manually drawn regions of interest (ROI). The counted pixel within the ROI were converted into volumes by timing the number of pixels by their individual volume. Afterwards, the tumor volume growth curves were fitted with an exponential function using the least squares methods; and then the time constant of the exponential was converted into a doubling time value to compare the two groups [17]. Finally, to assess survival, the body weight and mobility of each mouse were measured daily. Survival time was calculated from day 0 (tumor inoculation) to the day of sacrifice when experimental limit points were reached. 


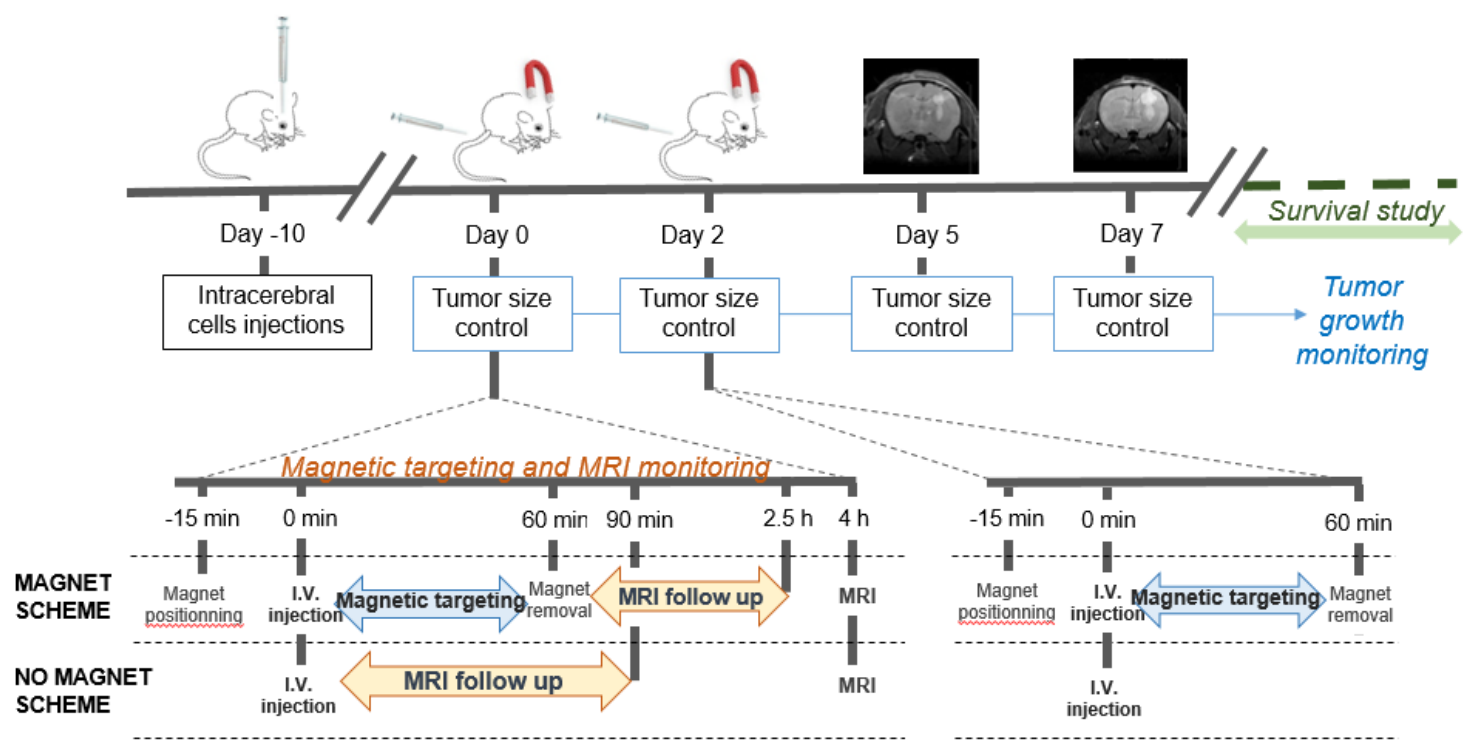

Fig. 1: Diagram of the in vivo studies performed.

\section{Statistical analysis}

All results are expressed as mean \pm standard deviation (SD). To demonstrate statistical differences, two-way ANOVA, unpaired twotailed t-test or log-Rank (mantel-Cox) survival tests were performed, using the software GraphPad Prism 5 for Windows.

\section{RESULTS AND DISCUSSION}

\section{SPION-DOX PNP characterization}

As previously described [10], the NP developed had a uniform size distribution (Fig 2a). The mean size was $227.4 \pm 18.2 \mathrm{~nm}$ by DLS (PDI: $0.066 \pm 0.021)$ and $116.2 \pm 37.4 \mathrm{~nm}$ measured on TEM images (size of minimum 150 NP measured on TEM images). A representative TEM image is presented in Fig $2 \mathrm{~b}$. A PDI less than 0.1 indicates that the sample consisted of a single size mode, without aggregates. Moreover, the zeta-potential value $(-14 \mathrm{mV})$ ensures that the PNP will repel each other and avoid aggregation. On the other hand, the observed differences in size between DLS and TEM may arise from the drying process and the subsequent shrinkage that polymeric chains underwent during TEM sample preparation $[10,19]$, as well as, to the different size-measuring method itself [20]. In this context, the amount of Fe and DOX encapsulated in SPION-DOX PNP was $5.7 \pm 0.6 \mu \mathrm{g} / \mathrm{mg}$ of DOX and $19.0 \pm 1.6 \mu \mathrm{g} / \mathrm{mg}$ of $\mathrm{Fe}$, in both cases corresponding to more than $80 \%$ encapsulation efficacy.

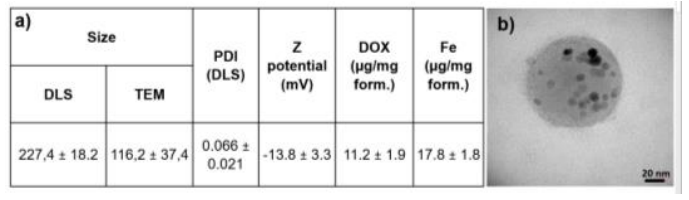

Fig. 2: a) Characterization of SPION-DOX PNP: size, polydispersity $(P D I)$ and $Z$ potential measured by DLS; size of minimum $150 \mathrm{NP}$ measured on TEM images; and DOX and Fe loading inside the PNP. b) TEM image of a representative SPION-DOX PNP.

\section{In vivo studies}

\subsection{Magnetic targeting and MRI monitoring}

An orthotopic U87 glioma model in female nude mice was used to evaluate whether the magnetic field influenced or not the retention of the NP inside the tumor. For that, we compared by MRI the GBM deposition of non-targeted and magnetically-targeted SPION-DOX PNP (at 16 $\mathrm{mg} \mathrm{Fe} / \mathrm{kg}$ body weight).

\section{Continuous monitoring}

As can be seen in Fig 3, magnetically-targeted NP were more likely to be retained in the tumor area. However, as revealed by the increase in R2* (Fig 3c), non-targeted NP could also be detected in the tumor tissues immediately after injection; however, 60 minutes later this contrast change induced by the presence of NP has disappeared (Fig 3a and c). Interestingly, targeting the NP with an external magnet for one 
hour led to a significant accumulation at the tumor level that lasted throughout the $2.5 \mathrm{~h}$ experimental time (Fig 3b). This accumulation was quantified by $\mathrm{R} 2 *$ relaxation calculation in the tumor and the non-tumor bearing hemisphere

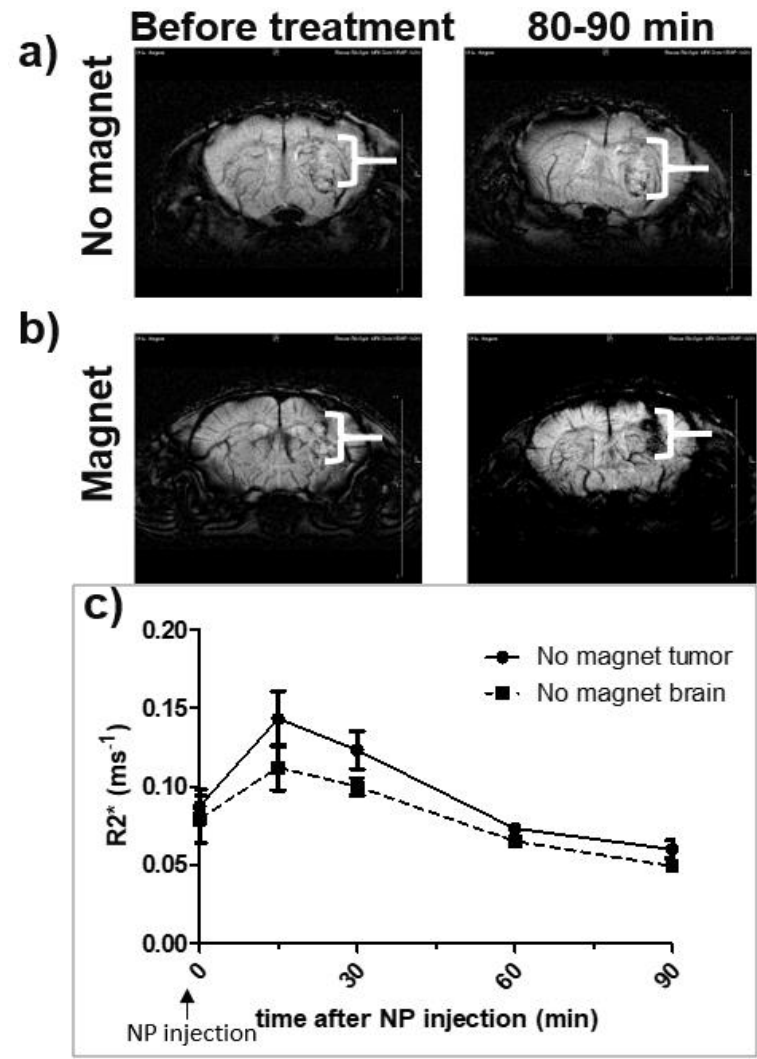

of mice (Figs 3c and e). In mice treated with NP but not targeted using magnets (Fig 3c) we observed that during the first minutes after administration the tumor tissue had a higher R2*
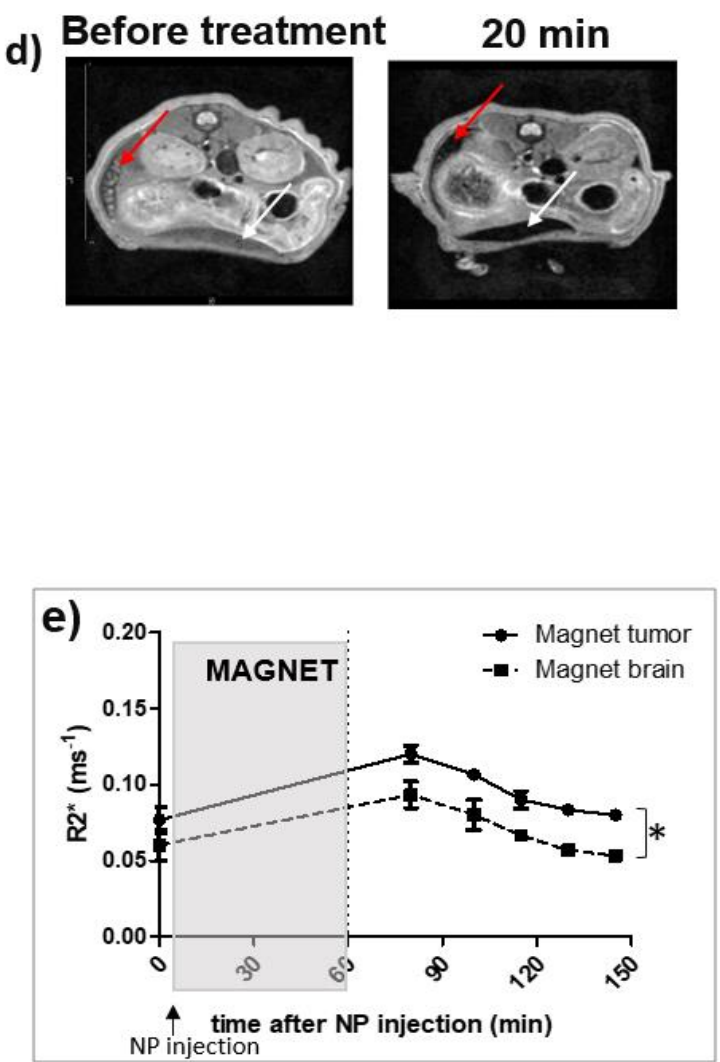

Fig. 3: NP brain uptake evaluation in tumor-bearing mice. a) Brain SWI images before and 80 minutes after i.v. injection of SPION-DOX PNP. b) Brain SWI images before and 90 minutes after i.v. injection of magnetically targeted SPION-DOX PNP. c) Relaxation rate $(R 2 *)$ in tumor and non-tumor tissue after i.v. injection of SPION-DOX PNP $(n$ $=3)$. d) Body transverse T2 images before and after i.v. injection of SPION-DOX PNP $(n=1)$. e) Relaxation rate $(R 2 *)$ in tumor and non-tumor tissue after i.v. injection of magnetically targeted SPION-DOX PNP; significant differences detected (statistical study ANOVA 2 tails $(P=0.0017)$ ). White brackets indicate the tumor location, plain white arrow the liver and dashed white arrow the spleen.

than the control brain; however, after one hour both had the same value. The higher vascularity of the tumor could explain this greater contrast in the short term: NP are in the bloodstream and are rapidly cleared by the mononuclear phagocyte system (MPS). This was confirmed by acquiring transversal T2 images over the liver and spleen of one mouse that revealed a high contrast and therefore accumulation of SPION-DOX PNP (Fig 3d). A closer analysis of R2* curves over time (Fig 3e) showed that 15-20 min after magnet removal (but 75-80 minutes after NP injection), the $\mathrm{R} 2 *$ of both the tumor and the control tissues was still increased as compared to the values measured before injection. This indicates that the magnet retained the SPION-DOX PNP throughout the entire brain area. Meanwhile, once the NP of the cerebral bloodstream receded, SPION-DOX PNP extravasation was observed only at the tumor region, with the $\mathrm{R} 2 *$ value in the healthy region decreasing rapidly whereas the $\mathrm{R} 2 *$ in the tumor area remained higher for the entire experimental time. This sustained difference between both tissues during the entire experimental period was found to be highly significant in a two-tailed ANOVA statistical study ( $p=0.0017)$. As previously described in detail, it would be possible for a SPION-DOX PNP to cross the BBB after being trapped within its endothelial cells due to a SPION mutual dipole-dipole attraction developed in a continuous magnetic field, which would lead to NP internalization by the malignant cells [18]. 


\section{Monitoring at 4 hours}

To evaluate the long/median term retention of the NP in the GBM after one hour external magnetic targeting, SWI images were acquired 4 hours after i.v. injection in 3 mice (Fig 4). Results appeared heterogeneous as in one mouse the susceptibility effects of NP were still observed at the tumor site (Fig 3a), in another there was a slight susceptibility effect (Fig 4b) and in the third one, no effect was detected (Fig 4c). On the other hand, no contrast was detected in the nontargeted tumors after 4 hours $(n=3)$. Therefore, although 4 hours seemed to be the limit of NP retention when intravenous SPION-DOX PNP were directed to GBM with a limited one hour of magnet guidance, the magnet targeting could be adapted to each mouse response attaining an individualized therapy.

All the results thus demonstrate the effectiveness of magnetic targeting to enhance preferential accumulation of the SPION-DOX PNP within brain tumors in an amount properly perceptible by MRI, but not within the healthy cerebral areas. Moreover, this study establishes the effectiveness of SPION-DOX PNP as contrast agent in vivo as well as the usefulness of MRI in non-invasive in vivo monitoring. In the same way, a $30 \mathrm{~min}$ or $1 \mathrm{~h}$ exposure to external magnet was shown to target starch coated nanocarriers magnetic nanocarriers to orthotopic tumors in a 9L-glioma bearing rat [21] as well as in mice bearing subcutaneous 9L-glioma tumors respectively.[22].

a)
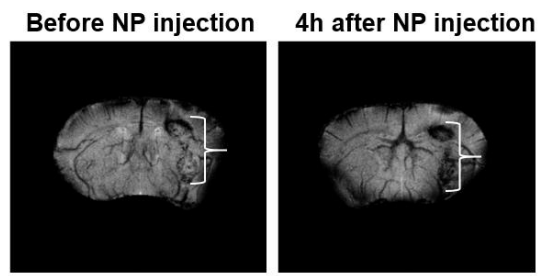

b)
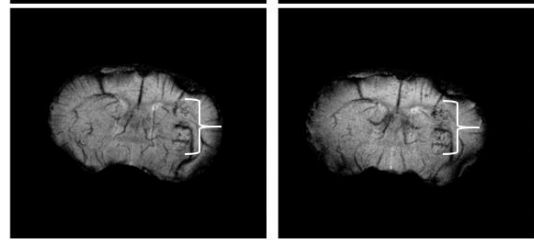

C)
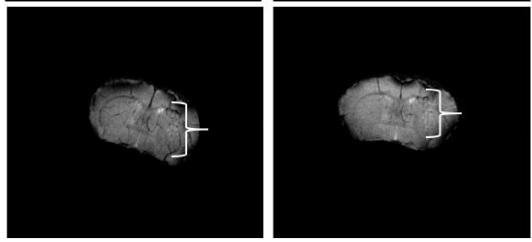

Fig. 4: SWI images of 3 magnet-exposed GMB before and 4 h after i.v. injection of SPION-DOX PNP. White brackets indicate tumor location.

\subsection{Efficacy studies}

The therapeutic effectiveness of SPION-DOX PNP magnetically targeted to the brain in an orthotopic U87 nude mice was evaluated. The final goal was to investigate whether magnetic targeting could further improve the access of PNP-entrapped DOX to the tumor and tumor growth inhibition as well as survival rates. For that, SPION-DOX PNP (at $5 \mathrm{mg}$ DOX/kg body weight) developed with the surfactant T80 were administered twice with a time interval of $48 \mathrm{~h}$ by intravenous injection. In one group the NP were targeted with the use of 1 hour of magnet $(n=7)$ and in a second group the NP were not targeted magnetically $(n=5)$.

\section{Tumor growth monitoring}

To assess treatment efficiency, tumor progression was monitored using T2-weighted MRI (Fig 5a and b), from which the tumor doubling time was calculated (Fig 5c). SPIONDOX PNP magnetically-targeted tumors were characterized by a $4.4 \pm 1.5$ days doubling time, whereas non-targeted tumors doubled in size in only $2.3 \pm 1.2$ days, being $2.93 \pm 0.77$ days [23] and $2.0 \pm 0.2$ days [17] the tumor doubling time for untreated mice in previous studies. Besides, the slower growth of the tumor when treated with magnetically-targeted SPION-DOX PNP was significantly different (two-tailed unpaired t-test study $(\mathrm{P}<0.05))$. These results indicated a positive therapeutic effect on the tumor as well as suggesting the enormous potential of the magnetically targeted-SPION-DOX PNP to deliver DOX to glioma cells, as free DOX displays very poor penetration into gliomas and cannot gain access to brain tumor cells [24]. Therefore, like other authors [25], we demonstrate therapeutic benefits based on the superparamagnetic characteristics of SPION that allow guidance by an external magnet and simultaneously provide contrast in MRI.

\section{Survival rate}

The overall median survival time was nonstatistically significantly improved by the therapeutic scheme used even though a light tendency is observed (Fig 5d) (31 vs 28 days, p $=0.0962)$. Despite the dosage of DOX $(5 \mathrm{mg} / \mathrm{kg}$; 2 doses) is within what is usually tested in glioma therapy in mice $(1-10 \mathrm{mg} / \mathrm{kg})$, more than 2 doses are often administered [27-33]. Moreover, the therapeutically scheme implied a first injection 10 days post cell inoculation and a second one 12 days post inoculation, meaning that the survival was evaluated at about 2 weeks after the end of the therapy, definitely enough time to let the fast growing U87 cells [17] to give rise to a deadly tumor. Therefore, checking the therapy impact 

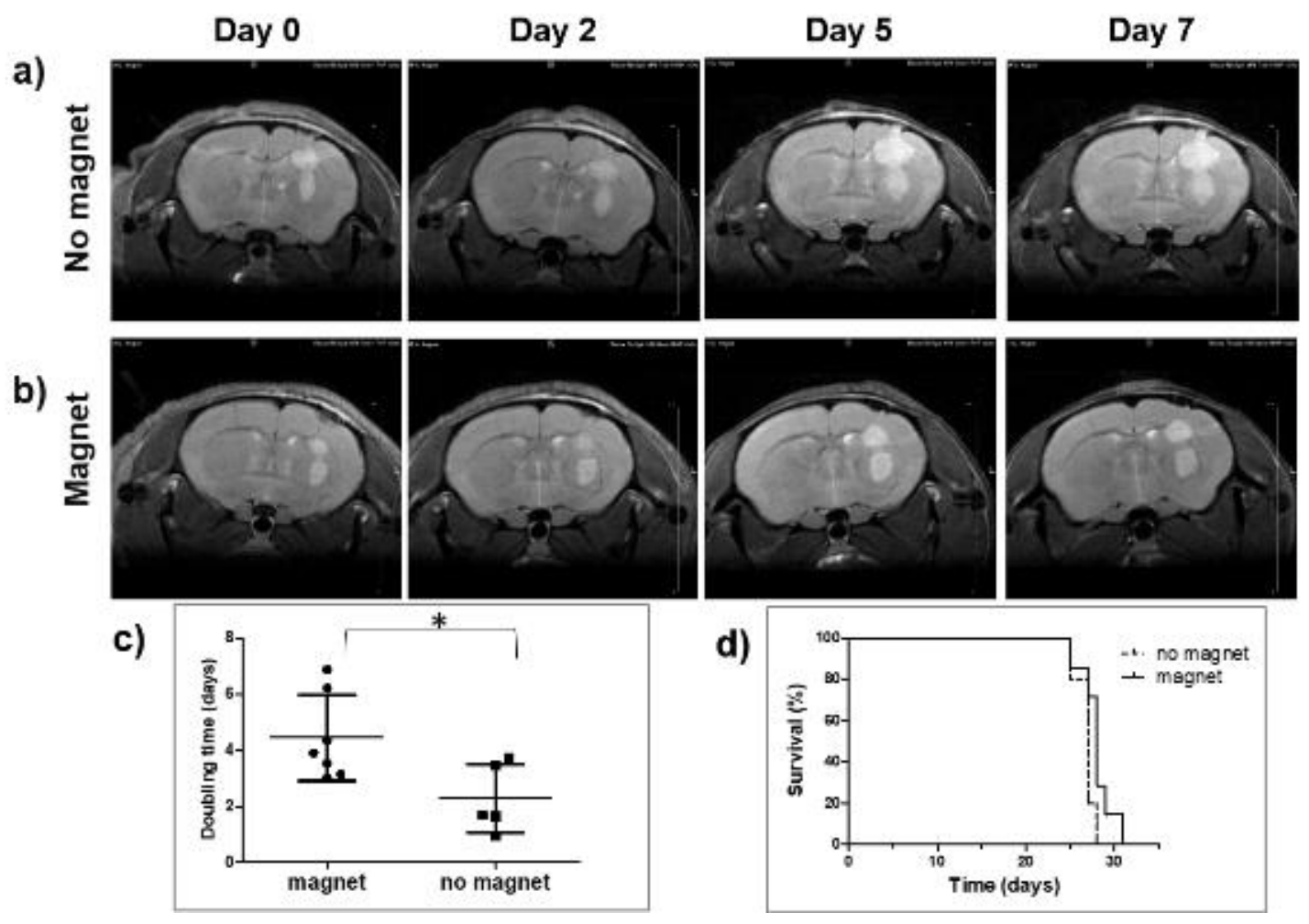

d)

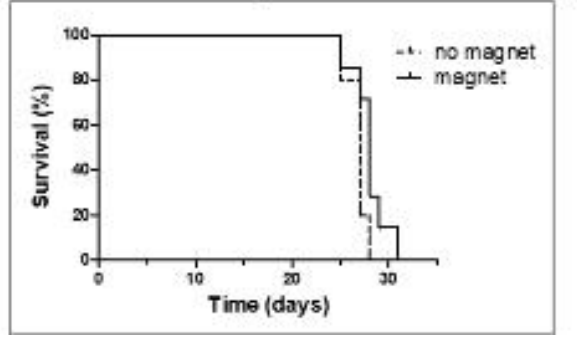

Fig. 5: Tumor bearing mice brains intravenously treated with SPION-DOX PNP (2 times, in alternative days with $(n=7)$ or without $(n=5)$ magnetic targeting. $a, b)$ T2-weighted images on days $0,2,5$ and 7 after the first injection c) Time needed for the tumors to double their size $(P<0.05)$. D) Survival rates.

through mice survival is not enough, and the follow-up of tumor growth profile in the short term (1 week) post might be a better indicator of the therapeutic scheme.

In a recent study, Ucakar B. et al. [34] found no significant difference between the brain accumulation of T80-coated and uncoated SPION-paclitaxel PNP in an orthotopic GBM model. However, in a subsequent study by the same authors [34,35], the magnetic targeting was found to enhance the brain accumulation of these $\mathrm{NP}$, their antitumor efficacy and the survival rate. A significant prolongation in the survival rates of mice was obtained using a longer targeting time (4h vs. 1h), a stronger magnet (1.4 T vs. $0.4 \mathrm{~T})$ and a larger number of doses (6 vs. 2). In our study we demonstrate the theranostic efficacy of the NP developed, since they were monitored by MRI at the same time as they treated the tumors. Nevertheless, to improve the survival rates, a larger number of doses [27,32,33] or a longer magnet exposure [25,36] could be used to achieve a higher DOX concentration in the tumor.

\section{CONCLUSIONS}

The objective of this study was to evaluate the theranostic properties of newly developed
doxorubicin-SPION loaded polymeric nanoparticles coated with T80 in a glioblastoma context. Taking advantage of the magnetic properties of the SPION, a significant enhancement of SPION-DOX PNP retention in the tumor was obtained after their magnetic targeting, with a significant decrease in the growth rate of the tumors. Further in vivo studies are needed to better the posology required to enhance survival rates, but magnetically targeted SPION-DOX PNP have proven to be a promising drug delivery system to enhance DOX delivery to brain tumors while also allowing non-invasive MRI monitoring of NP distribution.

\section{ACKNOWLEDGEMENTS}

This work was supported by the Fundación Caja Navarra and Navarra Government [grant number 411001-41210-4800-322302]. The authors would like to thank Angers Hospital \& University Animal Facility (SCAHU), Plateforme de Recherche en Imagerie et Spectroscopie Multimodales (PRISM-IRMAngers) for providing access to their facilities. 


\section{REFERENCES}

[1]

S. De Vleeschouwer, Glioblastoma, Codon Publications, 2017. doi:10.15586/CODON.GLIOBLASTOMA.2 017.

[2] P.S. Gaikwad, R. Banerjee, Nanotechnologybased strategies as novel therapies in gliomas, Ther. Deliv. 9 (2018) 571-592. doi:10.4155/tde-2018-0022.

[3] E.V. Valenciano, D. De Fisiología, E. De Medicina, D. Universidad, D.C. Rica, C. Costarricense, D.S. Social, S. Jose, C. Rica, M. Ángel, E. Miranda, E. De Medicina, D. Universidad, D.C. Rica, C. Costarricense, D.S. Social, S. Jose, C. Rica, Importancia de Células Madre Tumorales y Cultivos de Neuroesferas en Neurooncología, Neuroeje. 25 (2012) 55-60.

[4] Y. Malinovskaya, P. Melnikov, V. Baklaushev, A. Gabashvili, N. Osipova, S. Mantrov, Y. Ermolenko, O. Maksimenko, M. Gorshkova, V. Balabanyan, J. Kreuter, S. Gelperina, Delivery of doxorubicin-loaded PLGA nanoparticles into U87 human glioblastoma cells, Int. J. Pharm. 524 (2017) 77-90.

doi:10.1016/J.IJPHARM.2017.03.049.

[5] J. Kreuter, D. Shamenkov, V. Petrov, P. Ramge, K. Cychutek, C. Koch-Brandt, R. Alyautdin, Apolipoprotein-mediated transport of nanoparticle-bound drugs across the blood-brain barrier., J. Drug Target. 10 (2002) $317-25$. doi:10.1080/10611860290031877.

[6] A.R. Neves, J.F. Queiroz, S. Reis, Braintargeted delivery of resveratrol using solid lipid nanoparticles functionalized with apolipoprotein E, J. Nanobiotechnology. (2016). doi:10.1186/s12951-016-0177-x.

[7] M. Pinzón-Daza, R. Garzón, P. Couraud, I. Romero, B. Weksler, D. Ghigo, A. Bosia, C. Riganti, The association of statins plus LDL receptor-targeted liposome-encapsulated doxorubicin increases in vitro drug delivery across blood-brain barrier cells., Br. J.

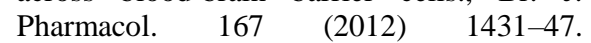
doi:10.1111/j.1476-5381.2012.02103.x.

[8] L. Battaglia, M. Gallarate, E. Peira, D. Chirio, E. Muntoni, E. Biasibetti, M.T. Capucchio, A. Valazza, P.P. Panciani, M. Lanotte, D. Schiffer, L. Annovazzi, V. Caldera, M. Mellai, C. Riganti, Solid Lipid Nanoparticles for Potential Doxorubicin Delivery in Glioblastoma Treatment: Preliminary In Vitro Studies, J. Pharm. Sci. 103 (2014) 2157-2165. doi:10.1002/jps.24002.

[9] C. Rousselle, P. Clair, J.M. Lefauconnier, M. Kaczorek, J.M. Scherrmann, J. Temsamani,
New advances in the transport of doxorubicin through the blood-brain barrier by a peptide vector-mediated strategy., Mol. Pharmacol. $57 \quad$ (2000) 679-86. http://www.ncbi.nlm.nih.gov/pubmed/10727 512 (accessed January 10, 2017).

[10] E. Luque-Michel, V. Sebastian, A. Larrea, C. Marquina, M.J. Blanco-Prieto, Coencapsulation of superparamagnetic nanoparticles and doxorubicin in PLGA nanocarriers: development, characterization and in vitro antitumor efficacy in glioma cells, Eur. J. Pharm. Biopharm. (2019). doi:10.1016/j.ejpb.2019.10.004.

[11] E. Luque-Michel, E. Imbuluzqueta, V. Sebastián, M.J. Blanco-Prieto, Clinical advances of nanocarrier-based cancer therapy and diagnostics., Expert Opin. Drug Deliv. 14 (2017) 75-92. doi:10.1080/17425247.2016.1205585.

[12] L.L. Israel, A. Galstyan, E. Holler, J.Y. Ljubimova, Magnetic iron oxide nanoparticles for imaging, targeting and treatment of primary and metastatic tumors of the brain, J. Control. Release. 320 (2020) 4562. doi:10.1016/j.jconrel.2020.01.009.

[13] W. Bin Shen, P. Anastasiadis, B. Nguyen, D. Yarnell, P.J. Yarowsky, V. Frenkel, P.S. Fishman, Magnetic enhancement of stem cell-targeted delivery into the brain following MR-guided focused ultrasound for opening the blood-brain barrier, Cell Transplant. 26 (2017) doi:10.1177/0963689717715824.

[14] Y. Qiu, S. Tong, L. Zhang, Y. Sakurai, D.R. Myers, L. Hong, W.A. Lam, G. Bao, Magnetic forces enable controlled drug delivery by disrupting endothelial cell-cell junctions, Nat. Commun. 8 (2017) 15594. doi:10.1038/ncomms 15594 .

[15] J.S. Weinstein, C.G. Varallyay, E. Dosa, S. Gahramanov, B. Hamilton, W.D. Rooney, L.L. Muldoon, E.A. Neuwelt, Superparamagnetic Iron Oxide Nanoparticles: Diagnostic Magnetic Resonance Imaging and Potential Therapeutic Applications in Neurooncology and Central Nervous System Inflammatory Pathologies, a Review, J. Cereb. Blood Flow Metab. 30 (2010) 15-35. doi:10.1038/jcbfm.2009.192.

[16] H. Li, H. Jin, W. Wan, C. Wu, L. Wei, Cancer nanomedicine: mechanisms, obstacles and strategies, Nanomedicine. 13 (2018) 16391656. doi: $10.2217 / \mathrm{nnm}-2018-0007$.

[17] L. Lemaire, J. Nel, F. Franconi, G. Bastiat, P. Saulnier, Perfluorocarbon-Loaded Lipid Nanocapsules to Assess the Dependence of U87-Human Glioblastoma Tumor pO2 on In 
Vitro Expansion Conditions, PLoS One. 11 (2016) e0165479.

doi:10.1371/journal.pone.0165479.

[18] H. Marie, L. Lemaire, F. Franconi, S. Lajnef, Y.-M. Frapart, V. Nicolas, G. Frébourg, M. Trichet, C. Ménager, S. Lesieur, Superparamagnetic Liposomes for MRI Monitoring and External Magnetic FieldInduced Selective Targeting of Malignant Brain Tumors, Adv. Funct. Mater. 25 (2015) 1258-1269. doi:10.1002/adfm.201402289.

[19] E. Luque-Michel, A. Larrea, C. Lahuerta, V. Sebastian, E. Imbuluzqueta, M. Arruebo, M.J. Blanco-Prieto, J. Santamaria, A simple approach to obtain hybrid Au-loaded polymeric nanoparticles with a tunable metal load, Nanoscale. 8 (2016) 6495-6506. doi:10.1039/c5nr06850a [doi].

[20] K. Fischer, M. Schmidt, Pitfalls and novel applications of particle sizing by dynamic light scattering, Biomaterials. 98 (2016) 7991.

doi:10.1016/J.BIOMATERIALS.2016.05.00

[21] B. Chertok, A.E. David, Y. Huang, V.C. Yang, Glioma selectivity of magnetically targeted nanoparticles: A role of abnormal tumor hydrodynamics, J. Control. Release. 122 (2007) 315-323. doi:10.1016/j.jconrel.2007.05.030.

[22] J. Zhou, J. Zhang, W. Gao, Enhanced and selective delivery of enzyme therapy to 9Lglioma tumor via magnetic targeting of PEGmodified, $\quad \beta$-glucosidase-conjugated iron oxide nanoparticles, Int. J. Nanomedicine. 9 (2014) 2905-2917. doi:10.2147/IJN.S59556.

[23] L. Lemaire, F. Franconi, B. Siegler, C. Legendre, E. Garcion, In vitro expansion of U87-MG human glioblastoma cells under hypoxic conditions affects glucose metabolism and subsequent in vivo growth, Tumor Biol. 36 (2015) 7699-7710. doi:10.1007/s13277-015-3458-3.

[24] P.M. Peiris, A. Abramowski, J. Mcginnity, E. Doolittle, R. Toy, R. Gopalakrishnan, S. Shah, L. Bauer, K.B. Ghaghada, C. Hoimes, S.M. Brady-Kalnay, J.P. Basilion, M.A. Griswold, E. Karathanasis, Treatment of invasive brain tumors using a chain-like nanoparticle, Cancer Res. 75 (2015) 13561365. doi:10.1158/0008-5472.CAN-141540 .

[25] N. Schleich, C. Po, D. Jacobs, B. Ucakar, B. Gallez, F. Danhier, V. Préat, Comparison of active, passive and magnetic targeting to tumors of multifunctional paclitaxel/SPIOloaded nanoparticles for tumor imaging and therapy, J. Control. Release. 194 (2014) 8291. doi:10.1016/J.JCONREL.2014.07.059.

[26] A. Clavreul, E. Roger, M. Pourbaghi-
Masouleh, L. Lemaire, C. Tétaud, P. Menei, Development and characterization of sorafenib-loaded lipid nanocapsules for the treatment of glioblastoma, Drug Deliv. 25 (2018) $1756-1765$. doi:10.1080/10717544.2018.1507061.

[27] Z. Sun, X. Yan, Y. Liu, L. Huang, C. Kong, $\mathrm{X}$. Qu, Application of dual targeting drug delivery system for the improvement of antiglioma efficacy of doxorubicin, Oncotarget. 8 (2017) 58823-58834.

[28] Y. Zhang, M. Zhai, Z. Chen, X. Han, F. Yu, Z. Li, X. Xie, C. Han, L. Yu, Y. Yang, X. Mei, Dual-modified liposome codelivery of doxorubicin and vincristine improve targeting and therapeutic efficacy of glioma, Drug Deliv. 24 (2017) 1045-1055. doi:10.1080/10717544.2017.1344334.

[29] Y. Li, L. Baiyang, B. Leran, W. Zhen, X. Yandong, D. Baixiang, Z. Dandan, Z. Yufu, L. Jun, Y. Rutong, L. Hongmei, Reductionresponsive PEtOz-SS-PCL micelle with tailored size to overcome blood-brain barrier and enhance doxorubicin antiglioma effect, Drug Deliv. 24 (2017) 1782-1790. doi:10.1080/10717544.2017.1402218.

[30] Z. Belhadj, M. Ying, X. Cao, X. Hu, C. Zhan, X. Wei, J. Gao, X. Wang, Z. Yan, W. Lu, Design of Y-shaped targeting material for liposome-based multifunctional glioblastoma-targeted drug delivery, J. Control. Release. 255 (2017) 132-141. doi:10.1016/j.jconrel.2017.04.006.

[31] Y. Fang, Y. Jiang, Y. Zou, F. Meng, J. Zhang, C. Deng, H. Sun, Z. Zhong, Targeted glioma chemotherapy by cyclic RGD peptidefunctionalized reversibly core-crosslinked multifunctional poly(ethylene glycol)- b poly( $\varepsilon$-caprolactone) micelles, Acta Biomater. $50 \quad$ (2017) 396-406. doi:10.1016/j.actbio.2017.01.007.

[32] S. Liu, Y. Guo, R. Huang, J. Li, S. Huang, Y. Kuang, L. Han, C. Jiang, Gene and doxorubicin co-delivery system for targeting therapy of glioma, Biomaterials. 33 (2012) 4907-4916. doi:10.1016/J.BIOMATERIALS.2012.03.03

[33] Y. Chen, Y. Huang, W. Liu, F. Gao, X. Fang, c(RGDyK)-decorated Pluronic micelles for enhanced doxorubicin and paclitaxel delivery to\&amp;nbsp;brain glioma, Int. J. Nanomedicine. $11 \quad$ (2016) 1629. doi:10.2147/IJN.S104162.

[34] B. Ucakar, N. Joudiou, J. Bianco, P. Danhier, F. Danhier, Magnetic targeting of paclitaxelloaded poly ( lactic- co -glycolic acid ) -based nanoparticles for the treatment of glioblastoma, Int. J. Nanomedicine. 13 (2018) 4509-4521. 
[35] L.P. Ganipineni, B. Ucakar, N. Joudiou, R. Riva, C. Jérôme, B. Gallez, F. Danhier, V. Préat, Paclitaxel-loaded multifunctional nanoparticles for the targeted treatment of glioblastoma, J. Drug Target. 27 (2019) 614623. doi:10.1080/1061186X.2019.1567738.

[36] W.-B. Shen, P. Anastasiadis, B. Nguyen, D. Yarnell, P.J. Yarowsky, V. Frenkel, P.S.
Fishman, Magnetic Enhancement of Stem Cell-Targeted Delivery into the Brain Following MR-Guided Focused Ultrasound for Opening the Blood-Brain Barrier, Cell Transplant. $26 \quad$ (2017) 1235-1246. doi:10.1177/0963689717715824. 